\title{
An Explanation of the Interactions between Waves and Electrons: Discussion of Electromagnetic Fields
}

\author{
Weiye $\mathrm{Xu}^{1, *}$ \\ ${ }^{1}$ Institute of Plasma Physics, Chinese Academy of Sciences, 230031 Hefei, China \\ *xuweiye@ipp.cas.cn
}

The interactions between waves and electrons are the basis of vacuum tubes and particle accelerators. In order to further understand the interactions, we proposed a new electromagnetic model. In this model, the photon is composed of two elementary particles - ephoton and mphoton, which are the basic particles that form the electric fields and magnetic fields, respectively. The calculation results show that the velocity of the ephoton is the same as the velocity of the electron in the conductor, which means that the ephotons are bound around the electrons. There are many ephotons around the electrons and the electrons can absorb the ephotons. This assumption does not conflict with existing experimental facts, nor does it conflict with existing theories such as the special theory of relativity. The proposed electromagnetic model can well explain the electromagnetic phenomena and the interactions between waves and electrons.

\section{Introduction}

The vacuum tubes [1], such as the traveling wave tubes, the back wave tubes, klystrons, magnetrons, gyrotrons, free electron lasers, etc. are used to generate high power microwaves, and are widely used in the fields such as the nuclear fusion, communication, radar, industrial microwave heating, etc. Particle accelerators [2] are the key devices for studying high-energy physics. The interactions between waves and electrons are the basic interactions in the vacuum tubes and the particle accelerators. Now using Maxwell's equations, the Lorentz force formula, and the energy conservation law, we can well analyze the interactions between the electromagnetic waves and the electrons. But that is just one of the correct ways to describe it. We do not really know why electrons and electromagnetic waves can transfer energy to each other. In this paper, we try to offer a new way of thinking in order to understand the interactions. Firstly, we discuss what is the electromagnetic field. A new electromagnetic model is proposed. Then we try to give the explanation of the interactions between waves and electrons according to the proposed electromagnetic model.

\section{Discussion of the electromagnetic fields}

Maxwell's equations do follow from the laws of electricity combined with the theory of special relativity [3]. There is a view that there is no such thing as a magnetic field, what appears to be a magnetic field is just the changing electric field. The theory of special relativity can explain the relationship between the electric field and the magnetic field. There is also a possibility that the theory of special relativity can only explain the relationship between the electric field and the magnetic field, but can not explain that they are the same thing. From a practical perspective, we can consider that both electric fields and magnetic fields exist.

We assume that there are two kinds of virtual photons, one is ephoton (short for electric-photon), the other one is mphoton (short for magnetic-photon). The particle flow density of the ephotons is the electric field intensity $\boldsymbol{E}$, and the particle flow density of the mphotons is the magnetic induction $\boldsymbol{B}$. Similar to the law of conservation of electric charge, we also believe in the law of conservation 
of ephoton. On the contrary, we think that the mphoton is not conserved. Thus, we have,

$$
\begin{gathered}
\boldsymbol{E}=\rho_{e} \boldsymbol{v}_{\boldsymbol{e}}, \\
\oint_{S} \boldsymbol{E} \cdot d \boldsymbol{S}=-\int_{V} \frac{\partial \rho_{e}}{\partial t} d V .
\end{gathered}
$$

Where, $\boldsymbol{E}$ is the particle flow density of the ephotons, i.e., the electric field intensity; $\rho_{e}$ is the particle density of the ephotons.

$$
\boldsymbol{B}=\rho_{m} \boldsymbol{v}_{\boldsymbol{m}},
$$

$\oint_{S} \boldsymbol{B} \cdot d \boldsymbol{S}=\int_{V} \nabla \cdot \boldsymbol{B} d V=\int_{V}\left(\rho_{m} \nabla \cdot \boldsymbol{v}_{\boldsymbol{m}}+\left(\nabla \rho_{m}\right) \cdot \boldsymbol{v}_{\boldsymbol{m}}\right) d V$.

Where, $\boldsymbol{B}$ is the particle flow density of the mphotons, i.e., the magnetic induction; $\rho_{m}$ is the particle density of the mphotons.

Compare Eq. (2) with Gauss's law,

$$
\oint_{S} \boldsymbol{E} \cdot d \boldsymbol{S}=\frac{1}{\varepsilon} \int_{V} \rho d V,
$$

we can find the relationship between the particle density of the ephotons $\rho_{e}$ and the charge density $\rho$,

$$
\frac{\rho}{\varepsilon}=-\frac{\partial \rho_{e}}{\partial t} .
$$

That is, the charge density is actually the change of ephoton density with time.

Compare Eq. (4) with the divergence equation of magnetic field (assuming no magnetic charge exists),

$$
\oint_{S} \boldsymbol{B} \cdot d \boldsymbol{S}=0
$$

we can find that,

$$
\rho_{m} \nabla \cdot \boldsymbol{v}_{\boldsymbol{m}}+\left(\nabla \rho_{m}\right) \cdot \boldsymbol{v}_{\boldsymbol{m}}=0
$$

We know that the differential form of Maxwell's equations in the isotropic medium are,

$$
\begin{gathered}
\nabla \times \boldsymbol{E}=-\frac{\partial \boldsymbol{B}}{\partial t}, \\
\nabla \times \boldsymbol{B}=\mu \boldsymbol{J}+\mu \varepsilon \frac{\partial \boldsymbol{E}}{\partial t}, \\
\nabla \cdot \boldsymbol{E}=\frac{\rho}{\varepsilon}, \\
\nabla \cdot \boldsymbol{B}=0 .
\end{gathered}
$$

Bring Eq. (1) and Eq. (3) into Eq. (9) and Eq. (10), respectively,

$$
\begin{gathered}
\left(\nabla \rho_{e}\right) \times \boldsymbol{v}_{\boldsymbol{e}}+\rho_{e} \nabla \times \boldsymbol{v}_{\boldsymbol{e}}=-\frac{\partial \rho_{m}}{\partial t} \boldsymbol{v}_{\boldsymbol{m}}-\frac{\partial \boldsymbol{v}_{\boldsymbol{m}}}{\partial t} \rho_{m} \\
\left(\nabla \rho_{m}\right) \times \boldsymbol{v}_{\boldsymbol{m}}+\rho_{m} \nabla \times \boldsymbol{v}_{\boldsymbol{m}}=\mu \boldsymbol{J}+\mu \varepsilon \frac{\partial \rho_{e}}{\partial t} \boldsymbol{v}_{\boldsymbol{e}}+\mu \varepsilon \frac{\partial \boldsymbol{v}_{e}}{\partial t} \rho_{e}
\end{gathered}
$$

The above two equations and Eq. (6) and Eq. (8) can be combined to obtain the following equations,

$$
\begin{gathered}
\left(\nabla \rho_{e}\right) \times \boldsymbol{v}_{\boldsymbol{e}}+\rho_{e} \nabla \times \boldsymbol{v}_{\boldsymbol{e}}=-\frac{\partial \rho_{m}}{\partial t} \boldsymbol{v}_{\boldsymbol{m}}-\frac{\partial \boldsymbol{v}_{\boldsymbol{m}}}{\partial t} \rho_{m}, \\
\left(\nabla \rho_{m}\right) \times \boldsymbol{v}_{\boldsymbol{m}}+\rho_{m} \nabla \times \boldsymbol{v}_{\boldsymbol{m}}=\mu \varepsilon \frac{\partial \boldsymbol{v}_{e}}{\partial t} \rho_{e}, \\
\boldsymbol{J}=-\varepsilon \frac{\partial \rho_{e}}{\partial t} \boldsymbol{v}_{\boldsymbol{e}}=\rho \boldsymbol{v}_{\boldsymbol{e}}, \\
-\frac{\partial \rho_{e}}{\partial t}=\frac{\rho}{\varepsilon}, \\
\rho_{m} \nabla \cdot \boldsymbol{v}_{\boldsymbol{m}}+\left(\nabla \rho_{m}\right) \cdot \boldsymbol{v}_{\boldsymbol{m}}=0 .
\end{gathered}
$$

The above five equations represent the motion of ephotons and the mphotons in the isotropic media. Similar to electromagnetic fields, the boundary relation of the ephotons and the mphotons are,

$$
\begin{gathered}
\mathbf{n} \times\left(\rho_{e 2} \boldsymbol{v}_{\boldsymbol{e} 2}-\rho_{e 1} \boldsymbol{v}_{\boldsymbol{e} \mathbf{1}}\right)=0, \\
\mathbf{n} \times\left(\frac{\rho_{m 2}}{\mu_{2}} \boldsymbol{v}_{\boldsymbol{m} \mathbf{2}}-\frac{\rho_{m 1}}{\mu_{1}} \boldsymbol{v}_{\boldsymbol{m} \mathbf{1}}\right)=\boldsymbol{\alpha}, \\
\mathbf{n} \cdot\left(\varepsilon_{2} \rho_{e 2} \boldsymbol{v}_{\boldsymbol{e} \mathbf{2}}-\varepsilon_{1} \rho_{e 1} \boldsymbol{v}_{\boldsymbol{e} \mathbf{1}}\right)=\sigma, \\
\mathbf{n} \cdot\left(\rho_{m 2} \boldsymbol{v}_{\boldsymbol{m} \mathbf{2}}-\rho_{m 1} \boldsymbol{v}_{\boldsymbol{m} \mathbf{1}}\right)=0,
\end{gathered}
$$

Where, $\boldsymbol{n}$ is the unit normal component of the interface, which is directed to the \#2 medium from the \#1 medium. The $\sigma$ is the surface density of the free charge. The $\alpha$ is the line density of the free current.

As can be seen from Eq. (17), the velocity of the ephoton is the same as the velocity of the charge (that is electron) in the conductor, which means that the ephotons are bound around the electrons.

In the vacuum, we assume that the ephoton density and the mphoton density are uniform. The Eq. (15) and Eq. (16) can be written as,

$$
\begin{gathered}
\rho_{e} \nabla \times \boldsymbol{v}_{\boldsymbol{e}}=-\frac{\partial \boldsymbol{v}_{\boldsymbol{m}}}{\partial t} \rho_{m}, \\
\rho_{m} \nabla \times \boldsymbol{v}_{\boldsymbol{m}}=\mu \varepsilon \frac{\partial \boldsymbol{v}_{e}}{\partial t} \rho_{e},
\end{gathered}
$$

From the above two equations, we can get the form of motion of ephotons and the mphotons. As shown in Fig. 1 (a), the ephoton moves at a constant velocity $v_{p}$ around the 
center of a sphere. The planes of motion of the two particles are perpendicular to each other. As shown in Fig. 1 (e) and (f), when the ephoton moves for a half cycle, it will change the direction of rotation. The ephoton and the mphoton will change the direction of movement, and the velocity remains constant. The pair of an ephoton and a mphoton make up a photon. The resonance frequency of the pair of an ephoton and a mphoton is the frequency of the phothon. The resonance radius is $\lambda$, i.e. the corresponding wavelength,

$$
\lambda=c / f \text {. }
$$

The actual velocity of the ephotons in the vacuum is,

$$
v_{p}=\pi c / 2 .
$$

The ephotons and the mphotons fill the entire universe. All the ephotons are always moving at speed $v_{p}$, but not the speed $c$. So, we predict that the transmission speed of the electric fields is $v_{p}$. The moving speed of the ephotons is a constant, and the propagating speed in the propagation direction changes periodically with an average value of $c$.

We think that the unit energy of the ephoton-mphoton pair is $h$, which is the Planck's constant, then the relativistic mass of the ephoton-mphoton pair is,

$$
m_{\gamma}=\frac{E}{c^{2}}=\frac{h}{c^{2}} .
$$

If the oscillation frequency of an ephoton-mphoton pair is $1 \mathrm{~Hz}$, the energy delivered in $1 \mathrm{~s}$ is $h$. If the oscillation frequency of an ephoton-mphoton pair is $v \mathrm{~Hz}$, the energy delivered in $1 \mathrm{~s}$ is $h v$, which is the photon energy that is currently widely believed. The higher the oscillation frequency of the ephoton-mphoton pair, the greater the energy transferred per unit time, so we usually think that the higher the frequency, the larger the photon energy. (a)

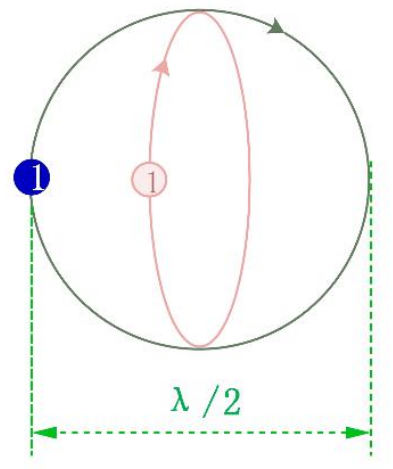

(c)

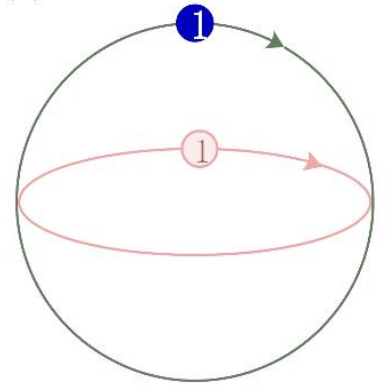

(e)

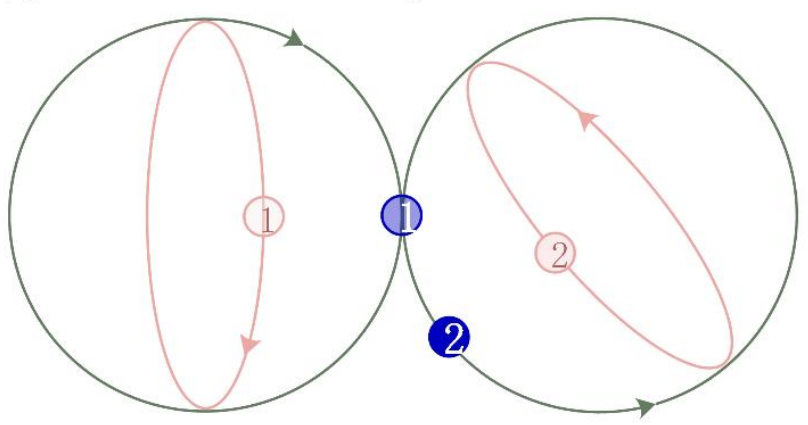

(g)

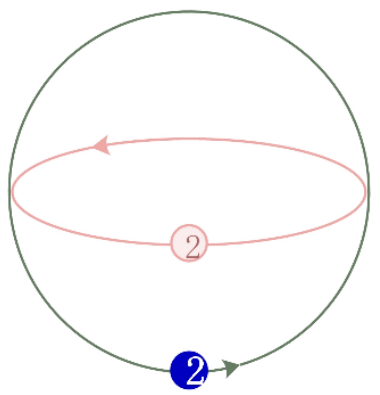

(d)

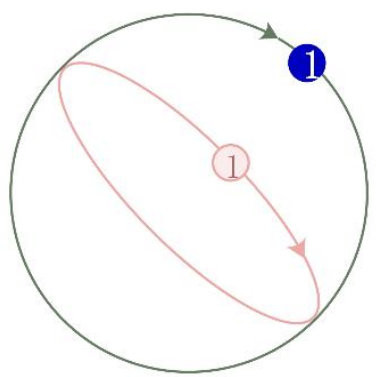

(f)

(h)

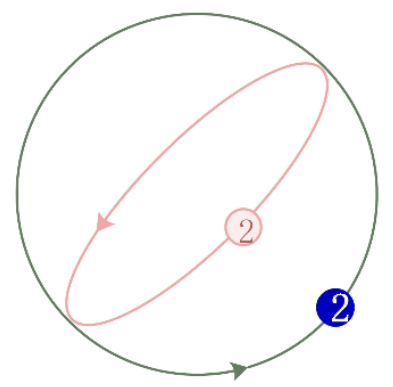

Fig. 1. The schematic diagram of the ephoton-mphoton particle pairs and the propagation of plane electromagnetic wave in vacuum (a) at the initial moment, (b) after one eighth of a cycle, (c) after a quarter of a cycle, (d) after three-eighth of a cycle, (e) after half a cycle, (f) after five-eighth of a cycle, (g) after three quarters of a cycle, and (h) after seven-eighth of a cycle. The red arrows in the figures are located on the inside of the paper.

There are some evidences show that the fine structure constant $\alpha$ may not be a constant $[4,5]$.

$$
\alpha=e^{2} / 4 \pi \varepsilon_{0} \hbar c .
$$

According to Albert Einstein's special theory of relativity, the speed of light in the vacuum, $c$, is a constant, and we 
believe that is true. And we believe $\hbar=h / 2 \pi$, is a constant, too. So we think that the charge of the electron, $e$, may be variable. The charge of the electron is not a Lorentz invariant, this is not contrary to the law of conservation of charge [6].

There is a possibility that the charges of positrons may not be exactly opposite to those of electrons [7-9]. In Ref. [7], the difference is measured using the measurements of Rydberg-energy and cyclotron-frequency ratios, which is,

$$
\left|q_{e^{+}}+q_{e^{-}}\right| / e<4 \times 10^{-8}
$$

There is a possibility that the mass of positrons may not be exactly equal to that of electrons $[10,11]$.

Based on Eq. (17) and the facts mentioned above, we proposed a model that is shown in Fig. 2, in which, there are many ephotons around the electrons and the positrons, and the electrons can absorb the ephotons, the positrons can release the ephotons. Although the electrons always absorb the ephotons, it always move in high speed (around the nucleus inside the atoms) with releasing the ephotons. The absorption rate and release rate are almost equal, so that the mass of the electrons change little.

Until now, no magnetic charge has been found yet [12]. Unlike the electrons that can absorb the ephotons, we think that there are no paticles that can absorb the mphotons. But there are a lot of mphotons in the universe. And the mphotons can be attracted by the ephotons to form the ephoton-mphoton particle pairs as shown in Fig. 1. That is the reason the electromagnetic waves can be generated.

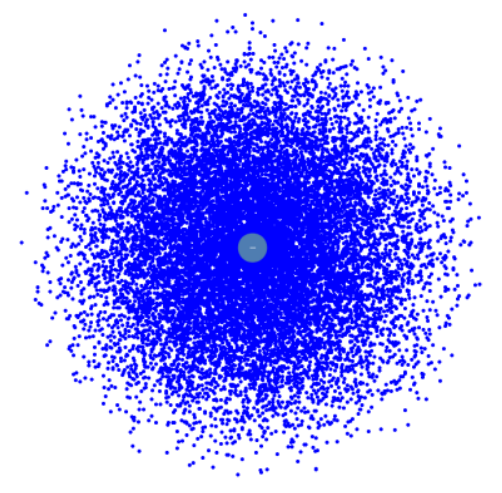

Fig. 2. The model of the electron.

The proposed model can easily explain many electromagnetic phenomena. For example, we can explain why electrons have magnetic moment. In order to maintain stability, electrons must spin. The electron spin drives the ephoton around the electrons to move circularly along the spin direction (motion in the z-y plane shown in Fig. 3 (a)), thereby generating a magnetic field and generating a magnetic moment.

As shown in Fig. 3 (b), when the electrons move in the $\mathrm{z}$ direction, more ephotons will cluster in the front, resulting in a magnetic field in the $\mathrm{x}-\mathrm{y}$ plane (perpendicular to the direction of motion). That is why the DC current can generate a stable magnetic field.
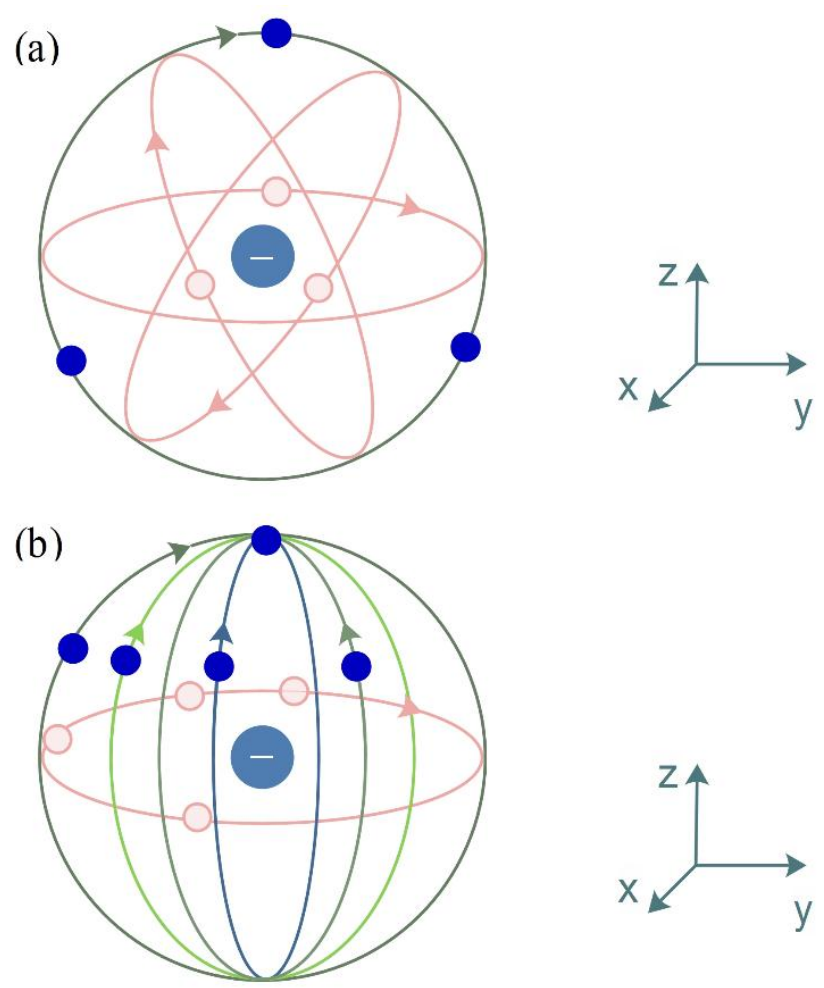

Fig. 3. Particle motion model around the electron when (a) the electron just spin without any axial movement, and (b) the electron moves along the z-direction.

The electron orbital magnetic moment and the nucleus spin magnetic moment have the same mechanism of generation as the electron spin magnetic moment. The molecular magnetic moment is constituted by the electron orbital magnetic moment, the electron spin magnetic moment, and the nucleus spin magnetic moment. The magnetization of the magnetic medium is a result of external magnetic field effects the magnetic moment of the molecule. 


\section{Interaction between waves and electrons}

Some kinds of high-power microwave sources [1] have been developed for many years to generate coherent electromagnetic radiation using the interaction between the waves and the electrons. The waves and electrons will interact to produce coherent radiation when the resonance happens between the waves and the electrons, which is,

$$
\omega \cong k_{z} v_{z}+s \Omega \text {. }
$$

Where, $\omega$ and $k_{z}$ are the wave angular frequency and axial wavenumber, $v_{z}$ is the electron axial velocity and $s$ is the cyclotron resonance harmonic number $(s=1,2, \ldots)$.

For devices utilizing Cherenkov radiation, like the traveling wave tubes, the back wave tubes, etc., $s=0$. That is, $\omega \approx k_{z} v_{z}$, i.e., $v_{p} \approx v_{z}$, i.e., when the electron axial velocity close to the phase velocity of electromagnetic wave, the interaction happens between the waves and the electrons.

For devices utilizing bremsstrahlung radiation, like the gyrotrons [13], $k_{z}<\omega / \mathrm{c}, \Omega=\mathrm{e} B_{0} / m_{e}$, where $B_{0}$ is the magnetic induction of the center of the gyrotron, $m_{e}$ is the relativistic mass of electron. The resonance condition is, $\omega \approx \mathrm{s} \Omega$, i.e., when the electron cyclotron frequency close to the electromagnetic frequency, the interaction happens between the waves and the electrons, too.

For free electron lasers [14], $k_{z} \approx \omega / c, \Omega=k_{w} v_{z}=2 \pi v_{z} / \lambda_{w}$, where $\lambda_{w}$ is the period of a spatially varying undulator or wiggler magnetic field. The above two kinds of interactions happen at the same time.

When the electron axial velocity close to the axial phase velocity of electromagnetic wave, $\omega \approx k_{z} v_{z}$, i.e, $\omega / k_{z} \approx v_{z}$. For devices with $\omega / k_{z} \approx c$, the way to make the waveselectrons interactions is to accelerate electrons to speed $c$. Another way to make the waves-electrons interactions happen is to decrease the axial phase velocity of the electromagnetic waves using the slow wave structure. In the slow wave structure, the minimum inner diameter is close to (or less than) the half wavelength. When the ephotons hit the metal wall, they will be absorbed by the electrons in the metal wall, and the electrons will move to release the new ephotons. Because the electrons absorb ephotons and release ephotons slower than the speed of light, so the axial phase velocity of the electromagnetic waves in the slower wave structure will be slower than the velocity of the light. When $v_{p}=\omega / k_{z}=v_{z}$, and the electromagnetic waves and the electrons resonate. When the velocity of the electrons $v_{p}$ is a little faster than the phase velocity of the electromagnetic waves, the speed of electrons will decrease, so as to tend to return to the resonance state. We assume that the faster the electron speed, the more the number of ephotons around. It may be the reason why the greater the speed, the greater the quality according to the special theory of relativity. When electrons move in the opposite direction of the electric field, which is shown in Fig. 4 (a), the electrons will accelerate and therefore absorb more ephotons, that is the progress of the electromagnetic energy converts into electronic kinetic energy in the accelerators. When electrons move in the same direction of the electric field, which is shown in Fig. 4 (b), the electrons will slow down and therefore release more ephotons, that is the process of the electronic kinetic energy converts into the electromagnetic energy in some electric vacuum devices such as the traveling wave tubes.
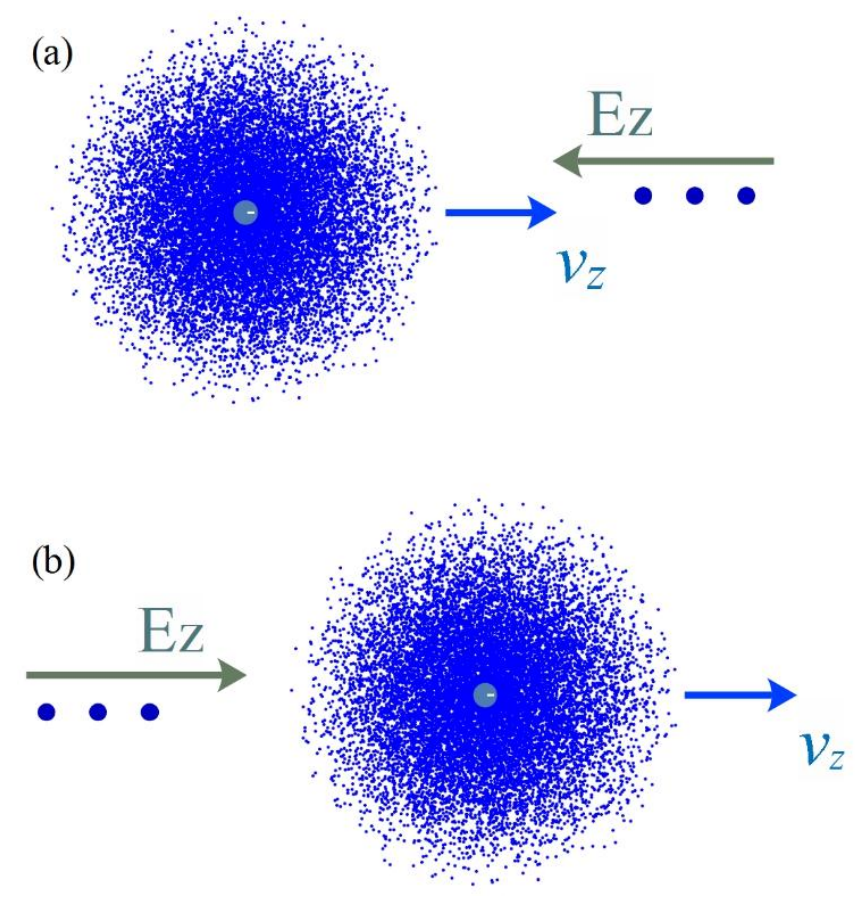

Fig. 4. Interactions between waves and electrons when (a) the direction of the electron movement is opposite to the electric field, (b) the direction of the electron movement is the same as the electric field.

When the electrons do uniform circular motion, the ephotons around the electrons will be thrown away. Due to inertia, the cyclotron frequency of the ephotons will be almost the same with the cyclotron frequency of the electrons. The cyclotron motion of the ephotons will attract 
the mphotons to form the ephoton-mphoton pairs, i.e. the photons, which are the basis particles of the electromagnetic wave. That is the interaction process between the waves and the electrons in the devices utilizing bremsstrahlung radiation.

\section{Discussion and conclusion}

A new electromagnetic model is proposed to explain the interactions between waves and electrons. In this model, the photon is composed of two elementary particles - ephoton and mphoton, which are the basic particles that form the electric fields and magnetic fields, respectively. There are many ephotons around the electrons and the electrons can absorb the ephotons. According to this model, the particle flow density of the ephotons is the electric field intensity $\boldsymbol{E}$, and the particle flow density of the mphotons is the magnetic induction $\boldsymbol{B}$. The charge density is actually the change of ephoton density with time. Using the ephotonmphoton pair assumption, a numerical algorithm may be developed in the future to simulate the propagation of the electromagnetic waves and the interactions between waves and electrons.

\section{References:}

[1] S. H. Gold and G. S. Nusinovich, Rev Sci Instrum 68, 3945 (1997).

[2] A. W. Chao and M. Tigner, Handbook of Accelerator Physics and Engineering (World Scientific, Singapore 1999).

[3] E. M. PURCELL and D. J. MORIN, Electricity and Magnetism (Cambridge University Press, 2013), Third edn.

[4] J. C. Pickering, A. P. Thorne, J. E. Murray, U. Litzén, S. Johansson, V. Zilio, and J. K. Webb, Monthly Notices of the Royal Astronomical Society 319, 163 (2000).

[5] J. K. Webb, M. T. Murphy, V. V. Flambaum, V. A.
Dzuba, J. D. Barrow, C. W. Churchill, J. X. Prochaska, and A.

M. Wolfe, Phys Rev Lett 87, 091301 (2001).

[6] Xiaobai Ai, Physics 26, 244 (2000).

[7] R. J. Hughes and B. I. Deutch, Phys Rev Lett 69, 578 (1992).

[8] B. Müller and M. H. Thoma, Phys Rev Lett 69, 3432 (1992)

[9] A. Schäfer and J. Reinhardt, Physical Review A 51, 838 (1995).

[10] M. S. Fee, S. Chu, A. P. Mills, R. J. Chichester, D. M. Zuckerman, E. D. Shaw, and K. Danzmann, Physical Review A 48, 192 (1993).

[11] S. Chu, A. P. Mills, and J. L. Hall, Phys Rev Lett 52, 1689 (1984)

[12] D. Milstead and E. J. Weinberg, Magnetic Monopoles, 2017.

[13] G. S. Nusinovich, M. K. A. Thumm, and M. I. Petelin, J Infrared Millim Te 35, 325 (2014).

[14] T. Manfred, State-of-the-Art of High Power Gyro-Devices and Free Electron Masers, Report No. KITSR 7575, 2010.

\section{Acknowledgments:}

We are grateful to Huayu Li for helpful discussions.

\section{Postscript:}

Since electrons can absorb ephotons and simultaneous release of ephotons due to spin, a stable equilibrium state can be achieved. Protons and positrons are unstable due to the emission of ephotons and the simultaneous release of ephotons due to spin. We have a bold speculation that electromagnetic interactions and weak interactions are caused by the absorption or emission of ephotons, and substances that cannot absorb or emit ephotons are so-called dark matter. 